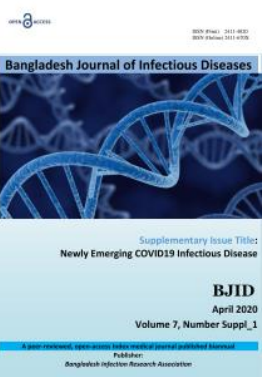

\title{
Diagnostic Value of High Resolution CT Imaging for COVID-19 in 24 Confirmed Cases
}

\author{
Sayed Abdulla Jami ${ }^{1}$, Liu Chang $\mathrm{Hao}^{2}$, Brotendu Shekhar Roy ${ }^{3}$, Zhanwen Zhou ${ }^{4}$, MM Sohel \\ Tanvir ${ }^{5}$, Siam Al Mobarak ${ }^{6}$
}

${ }^{1}$ Resident Doctor \& Research Assistant, Department of Spinal Surgery, General Hospital of Ningxia Medical University, Ningxia Medical University, Ningxia, China; ${ }^{2}$ Postgraduate \& Research Assistant, Department of Spinal Surgery, General Hospital of Ningxia Medical University, Ningxia Medical University, Yinchuan, Ningxia, China; ${ }^{3}$ Lecturer, Changsha Medical University, Changsha, Hunan, China; ${ }^{4}$ Resident Doctor \& Post Graduate, Department of Spinal Surgery, General Hospital of Ningxia Medical University, Ningxia Medical University, Yinchuan, Ningxia, China; ${ }^{5}$ Internship, Department of Spinal Surgery, General Hospital of Ningxia Medical University, Ningxia Medical University, Yinchuan, Ningxia, China; ${ }^{6}$ Postgraduate, First Affiliated Hospital of Guangxi Medical University, Nanning, Guangxi, China

[Received: 26 March 2020; Accepted: 2 April 2020; Published: 25 April 2020]

\begin{abstract}
Background: Covid19 virus causes pandemic worldwide. Objective: The purpose of the present study was to investigate the clinical features and imaging characteristics on chest High-Resolution Computer Tomography (HRCT) of COVID-19. Methodology: This retrospective study was conducted in the special designated department for covid-19 isolation unit under Ningxia Medical University from 10 January to 15 February 2020. The medical history, clinical symptoms, and imaging characteristics of confirmed COVID-19 patients from a single center were retrospectively analyzed. All cases underwent a chest HRCT examination. Results: A total number of 24 patients were recruited for this study of which 18 cases had clinical symptoms of respiratory tract infection; 2 cases had symptoms other than respiratory tract and 4 cases had asymptomatic. There were 15 males and 9 females aged 17 to 74 years. The main chest HRCT manifestation was diffuse ground-glass opacity (GGO) in the peripheral zone of the lungs and under the pleura. Pulmonary interstitial changes, bronchial air signs, bronchial vascular bundle thickening, paving stone signs, and "white lung" changes appeared in some cases. A few cases have enlarged lymph nodes and a small amount of pleural effusion. Conclusion: The COVID-19 has specific characteristics on chest HRCT. [Bangladesh Journal of Infectious Diseases, April 2020;7(suppl_1):S3-S10]
\end{abstract}

Keywords COVID-19; HRCT; Diagnosis; Imaging

Correspondence: Dr. Sayed Abdulla Jami, Department of Spinal Surgery, General Hospital of Ningxia Medical University, Ningxia Medical University 804 Shengli Street, Xingqing District, Yinchuan, 750004, Ningxia, People's Republic of China; Email: jami41@live.com; ORCID: 0000-0002-2479-2589; Cell no.: +008613259510600

Conflict of interest: All authors have no competing interests.

Funding agency: Authors received no financial support for research or publication of this article yet.

Contribution to authors: SAJ conceived and designed the study; SAJ, LCH, and BSR collected the data; ZZ, SAM \& MMST checkout the follow-up; SAJ \&LCH wrote different parts of the manuscript; SAJ \&BSR revised the manuscript. All authors approved the final manuscript. Contribute Equally as First Author: Sayed Abdulla Jami, Liu Chang Hao and Brotendu Shekhar Roy

How to cite this article: Jami SA, Hao LC, Roy BS, Zhou Z, Tanvir MMS, Siam Al Mobarak SA. Diagnostic Value of HighResolution CT Imaging for COVID-19 in 24 Confirmed Cases. Bangladesh J Infect Dis 2020;7(suppl_1):S3-S10

Copyright: $\odot 2020$. Jami et al. Published by Bangladesh Journal of Infectious Diseases. This article is published under the Creative Commons CC BY-NC License (https://creativecommons.org/licenses/by-nc/4.0/). This license permits use, distribution and reproduction in any medium, provided the original work is properly cited, and is not used for commercial purposes. 


\section{Introduction}

COVID-19, a new type of virus, caused acute respiratory infectious disease, mainly inflammatory lesions in the lungs caused by this virus and quickly spread to other parts of China and abroad ${ }^{1}$. A new coronavirus has been isolated from airway epithelial cells of infected persons through pathogenic laboratory analysis. The main transmission route is respiratory droplets and close contact or even a possibility of aerosol transmission and incidence rate is high ${ }^{2}$.

With the aggravation of the epidemic situation, the dynamic epidemiological history of patients became more and more obscure, and second-generation patients with the history of close contact related to covid-19 infected people living in the epidemic-free area are increasing. Due to the rapid increase in the number of patients, the shortage of nucleic acid detection reagents, and the existence of falsenegative nucleic acid results, imaging technology and especially HRCT are significant for the early diagnosis of diseases. This study had been reviewed the current confirmed case data of the hospital and analysis with the relevant literature of COVID-19 to summarize and to discuss the features and characteristics of the chest imaging of COVID-19 patients.

\section{Methodology}

General Information: This retrospective study was performed on the clinical characteristics and imaging findings of 24 patients diagnosed with COVID19 in the General Hospital of Ningxia Medical University, Yinchuan, Ningxia, China from $10^{\text {th }}$ January 2020 to $15^{\text {th }}$ February 2020 for a period of 1 months and 5 days. It was considered a suspected case, if the patients had met the following two conditions according to the "Diagnosis and Treatment Scheme of Novel Coronavirus Infection Pneumonia (Fifth Edition Trial)" ${ }^{13}$ : (1) epidemiological history: there was a history of travel or residence in Wuhan area or other areas of Hubei province fourteen days before the onset of the disease; Within 14 days prior to onset, contact with patients with fever or respiratory symptoms in Wuhan or other areas where transmission continues; aggressive cluster or epidemiological association of coronavirus infection. (2) Clinical manifestations are a fever with typical pneumonia imaging features; the total number of white blood cells is normal or reduced, or the number of lymphocytes is reduced in the early stage of onset. A confirmed case was referred to the detection of a novel coronavirus positive nucleic acid test by real-time fluorescent RT-PCR based on meeting the criteria for a suspected case. Nucleic acid tests were positive in 24 patients. Review board of university and hospital approval was obtained before starting research. The ethical standards of the responsible committee on human experimentation (institutional and national) and with the Helsinki Declaration of 1975, as revised in 2000 and 2008 were followed accordingly.

Equipment and Methods: PHILIPS Brilliance 64slice CT scanning device was used for highresolution CT scans of both lungs. The scanning range is from the entrance of the thorax to the bottom of the lungs. Scanning parameters: tube voltage $120 \mathrm{kV}$, tube current $50 \mathrm{~mA}$ and pitch is 1.0 ; $5 \mathrm{~mm}$ layer thickness, $5 \mathrm{~mm}$ layer spacing, matrix $512 \times 512$, scanning time $<5 \mathrm{~s}$; Reconstruction was performed using the bone algorithm with a thickness of $1 \mathrm{~mm}$. Preset lung window level -450 to $550 \mathrm{HU}$, window width -1300 to $-1500 \mathrm{HU}$; The preset mediastinal window is $30 \sim 45$, and width is 310 360 HU.

Image analysis: Two chief physicians reviewed HRCT images. Both had different opinions. Afterward, judged by another senior physician and the diagnosis was determined after discussion. The distribution of lesions was analyzed by multi-plane recombination and maximum intensity projection recombination imaging such as mediastinal lymph nodes and pleural effusion.

Statistical Analysis: SPSS v20.0 software was used for statistical analysis. The counting data were expressed as the number of cases or percentage. A total of 24 patient's data were included for analysis. Time and age intervals were regarded as continuous variables.

\section{Results}

Clinical Manifestation: There were 15 males and 9 females, ratio 5:3. The age ranged from 17 to 74 years old, with an average of $(53 \pm 13.0)$ years old. 17 cases were over 60 years old $(71 \%) ; 18$ cases had a history of staying in the affected area and a history of close contact with infected cases; 6 cases had no evident history of communication in the affected area. Among the 24 COVID-19 confirmed cases, there were 18 cases $(75 \%)$ respiratory symptoms with such as fever and cough, including 12 cases $(50 \%)$ of fever, 3 cases of simple cough $(12.5 \%)$, and 3 cases of a fever combined with cough $(12.5 \%)$. 
Table 1: The Following Assumptions Were Used While Generating the Estimates

\begin{tabular}{|l|c|}
\hline Assumptions & $\begin{array}{c}\text { Value } \\
\text { (Days) }\end{array}$ \\
\hline Mean incubation period & 5.1 \\
\hline Infectiousness starts & $\begin{array}{c}4.6 \\
\text { (after the } \\
\text { entry of the } \\
\text { virus) }\end{array}$ \\
\hline Mean generation time & 6.5 \\
\hline Basic reproduction number & 2.4 \\
\hline Symptomatic cases & $83.33 \%$ \\
\hline $\begin{array}{l}\text { Average duration between onset } \\
\text { of symptoms and hospitalization }\end{array}$ & 5 \\
\hline $\begin{array}{l}\text { Average length of hospital stays } \\
\text { for patients without a need for } \\
\text { critical care }\end{array}$ & 8 \\
\hline $\begin{array}{l}\text { Average length of hospital stays } \\
\text { for patients including with need } \\
\text { for critical care }\end{array}$ & 10.4 \\
\hline
\end{tabular}

There were 2 cases $(8 \%)$ of diarrhea and 4 cases (17\%) of asymptomatic. Laboratory results showed that the white blood cell count increased in 6 cases $(25 \%)$, was normal in 6 cases $(25 \%)$, and decreased in 12 cases $(50 \%)$.

Lymphocyte count increased in 5 cases $(21 \%)$ and decreased in 19 cases (79\%). Neutrophil cells increased in 10 cases (42\%), normal in 9 cases $(37 \%)$, and decreased in 5 cases $(21 \%)$. Elevated Creactive protein in all patients (100\%). Author had summarized the assumptions and were used while generating the estimates in covid-19 cases during research (Table 1).

Imaging Findings: Out of 24 patients 19(79.0\%) cases had lesions in the peripheral belt and subpleural areas of both lungs; $3(13.0 \%)$ cases showed in the upper lobes of both lungs, and $2(8.0 \%)$ cases had single lesions near the hilum. However, 22(92.0\%) cases had shown ground glass shadow in the lungs (GGO) (Figure 1).

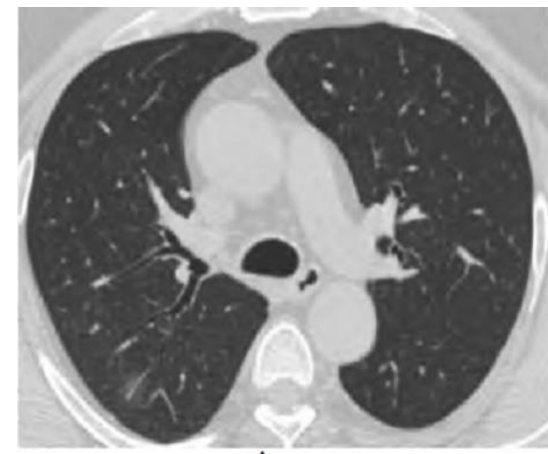

A

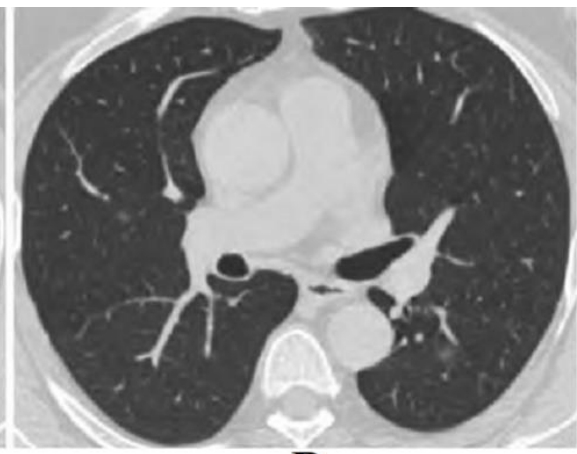

B

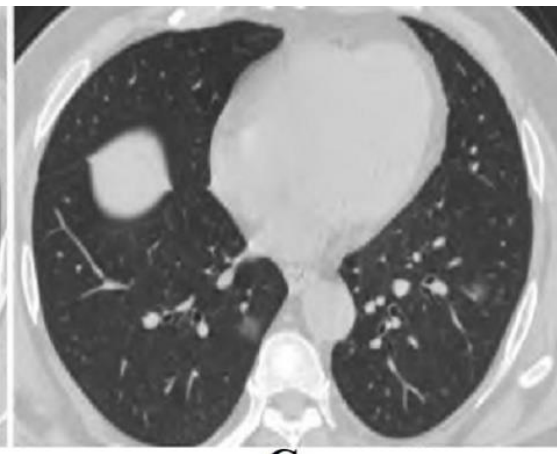

C

Figure I: The patient was a 39-year-old male with no fever, had a history of occupational exposure, and was admitted to the hospital 5 days later. On the day of admission, the patient underwent a chest HRCT examination. A-C is a plain CT scan of the lungs showing multiple small patchy GGOs in the lower lungs, blood vessels in the lower right lung lesions are slightly thickened, remaining lesions are clear, and the density is uniform.

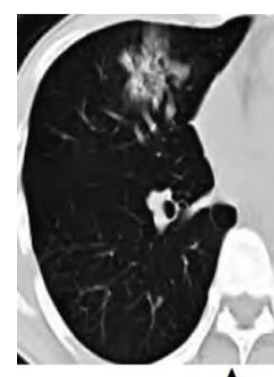

A

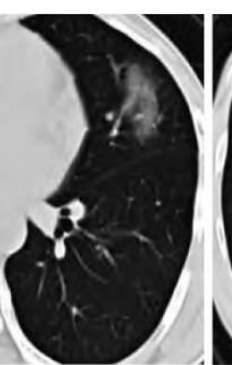

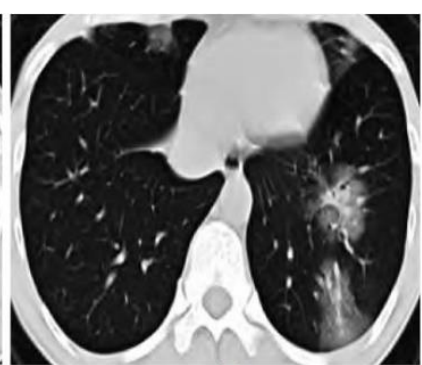

B

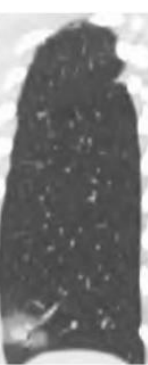

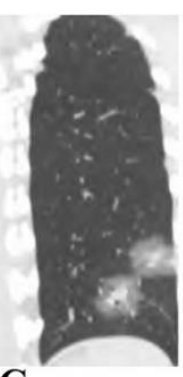

C

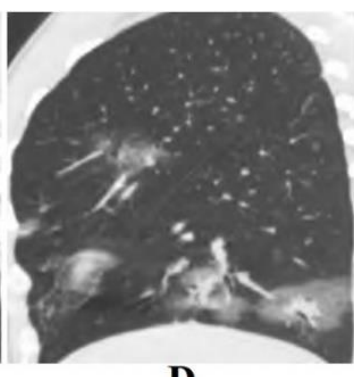

D

Figure II: The Patient was 34 years old female, history of living in affected areas. Cough and fever more than 1 week were admitted to the hospital on the day of the HRCT examination. A, B picture shows a cross-section image of a CT plain lungs; C, D respectively shows the reconstruction of the coronary and sagittal planes with plain CT scan of the lungs, Multiple laminar GGOs are shown in the two lungs, high density nodules can be seen in the lesions, the blood vessels in the lesions are thickened, structural disturbances can be seen as well 


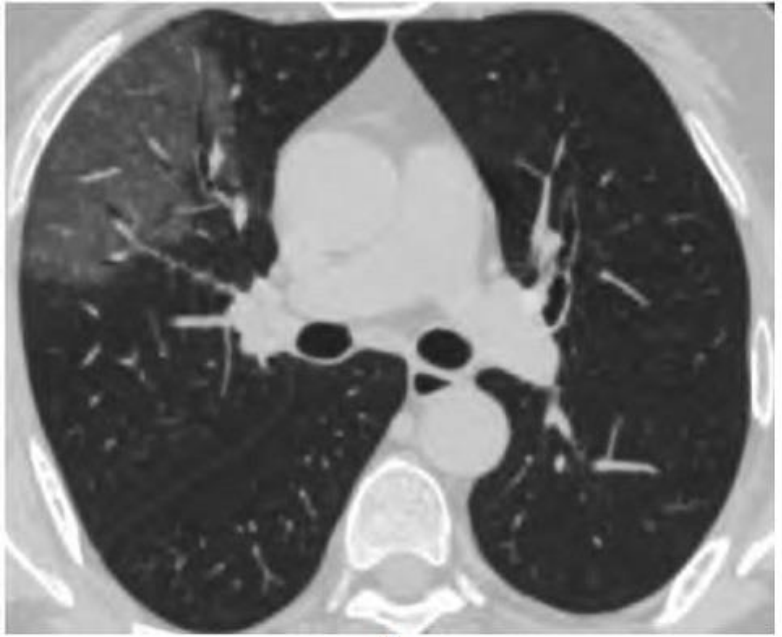

A

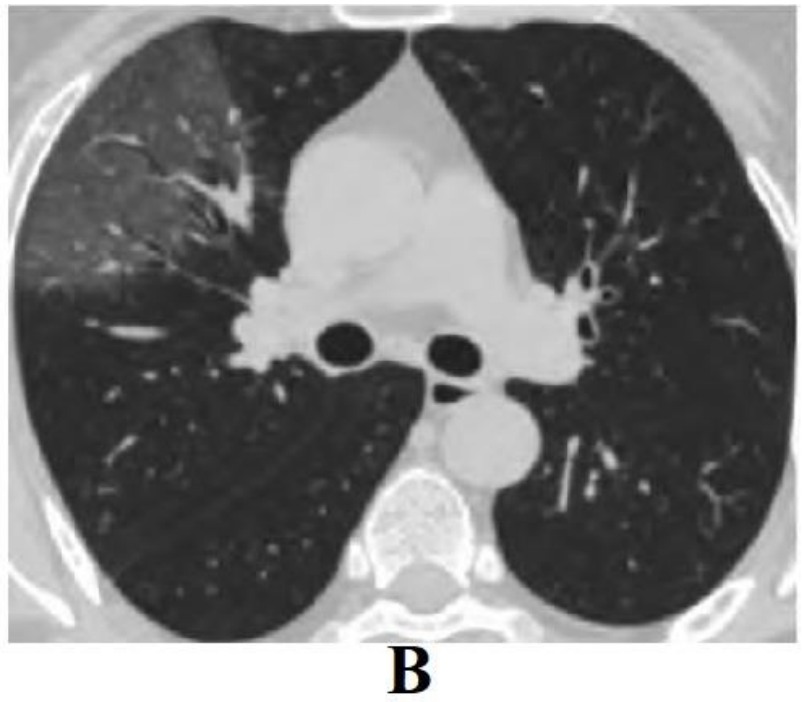

Figure III: The patient was 31 years old male. Live in the epidemic-free area, the history of fever and chest tightness was for 1 day, received HRCT examination on the day of admission. A and B CT scan image showing the wedgeshaped patches of GGO shadows in the right middle lung, thickening of small vessels in the lesion, and air bronchogram

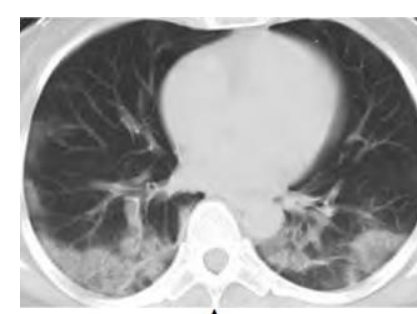

A

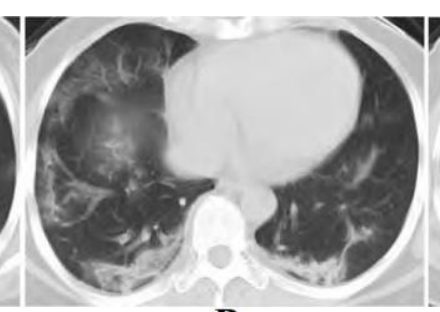

B

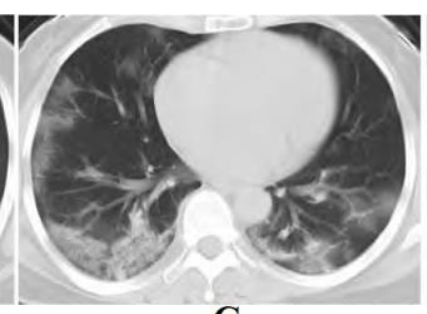

C

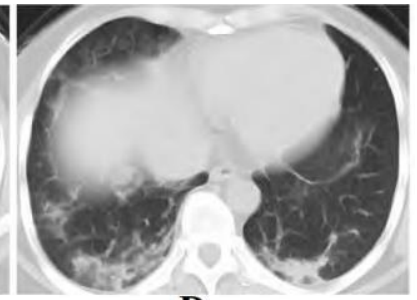

D

Figure IV: The patient was 29 years old female. A living history of close contact with an infected person. Had physical discomfort fever for 3days and received HRCT examination the next day of admission. A-D cross-sectional image of the lungs by plain CT scan showing patchy distribution of GGO shadow, vascular and bronchial bundle thickening in both lungs

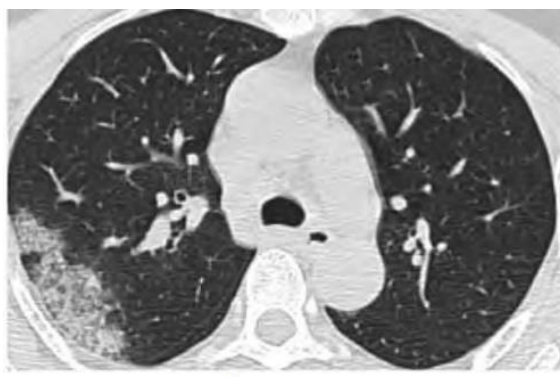

A

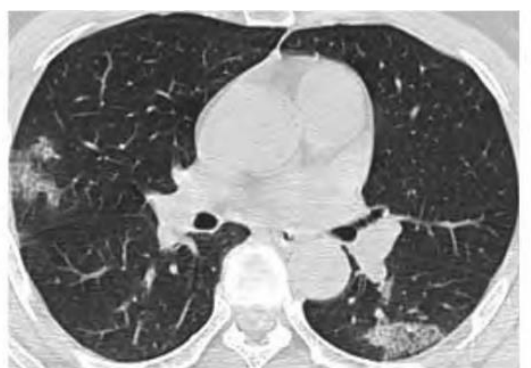

B

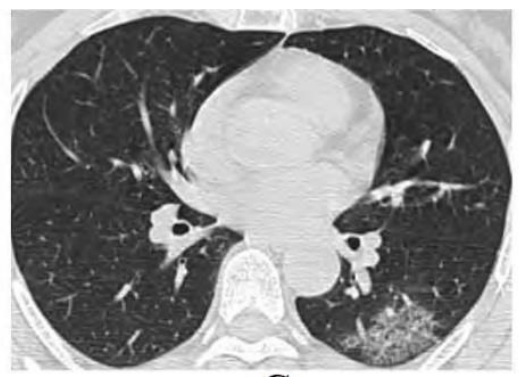

C

Figure V: The patient was 48 years old male. Have a history of close contacts. Had fever and cough for 3 days. Was admitted and HRCT examination done on 3rd day. A-C image plain lung CT scan showing multiple GGO lesions in both lungs, which are distributed in the peripheral zone of both lungs, and paving stones can be seen in the right lower lung lesions

GGO with solid nodules was seen in 14 cases (58\%) (Figure II). Broncho vascular thickening and air bronchogenic features were seen in the lesion (Figure III, IV, V). Besides, there were 8 cases (33\%) saw large-scale consolidation, 3 cases (13\%) were accompanied by a small amount of pleural effusion, and 2 cases (8\%) showed enlarged mediastinal or bilateral hilar lymph nodes. There were 2 cases $(8 \%)$ presented with "white lung" (Figure VI), and 7(29\%) cases presented with gridshaped fiber stripe shadow (Figure VII). 


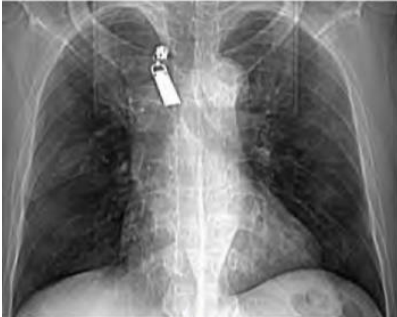

A

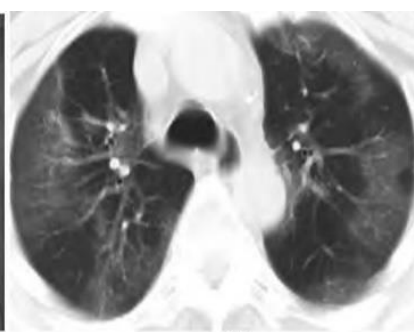

B

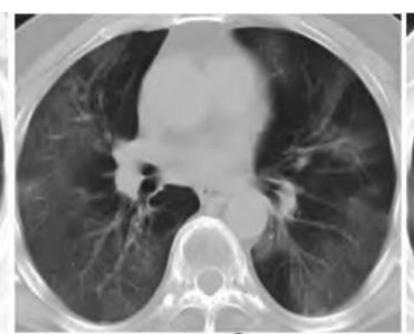

C

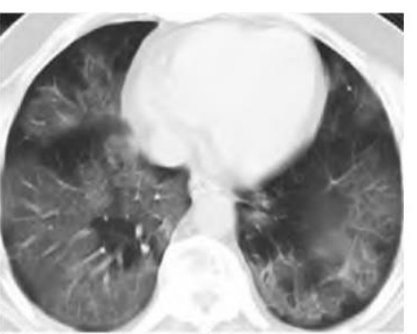

D

Figure VI: The patient was 55 years old male. No contact history of infected areas. Cough and sputum were accompanied by fever for 4 days. A chest DR examination was performed on the day of admission, and an HRCT examination was performed on the 3rd day. A: is a DR image showing the diffusely increased density of the two lungs; B-D is a CT image showing a diffuse large patchy GGO image of the two lungs, with a thickened bronchial vascular bundle, widened leaflet intervals, a grid Changes and typical "white lung" images.

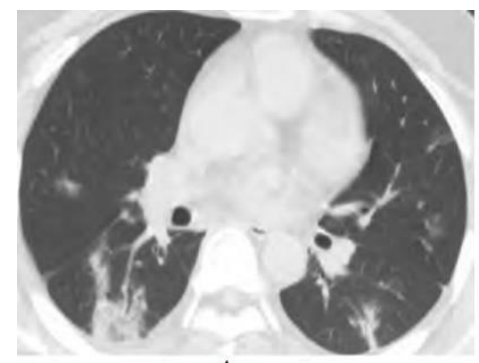

A

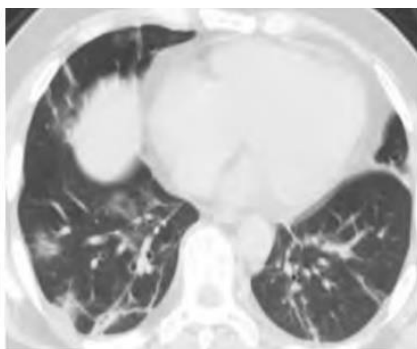

B

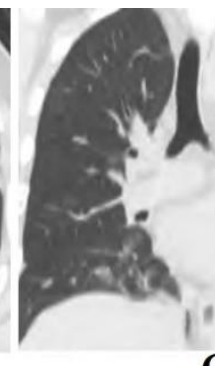

C

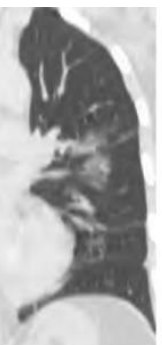

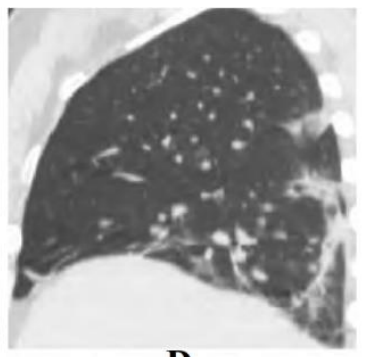

D

Figure VII: The patient was 55 years old female. There is a history of contact in the affected areas. HRCT examination was reviewed at 10 days after treatment. A and B are cross-sectional images of a plain CT lung window; C and D respectively showed the coronal plane and sagittal plane images reconstructed in the lung window, multiple patchy GG0 shadows, and stripy high-density shadows in both lungs, and thickening of the bronchiolar wall in some lesions

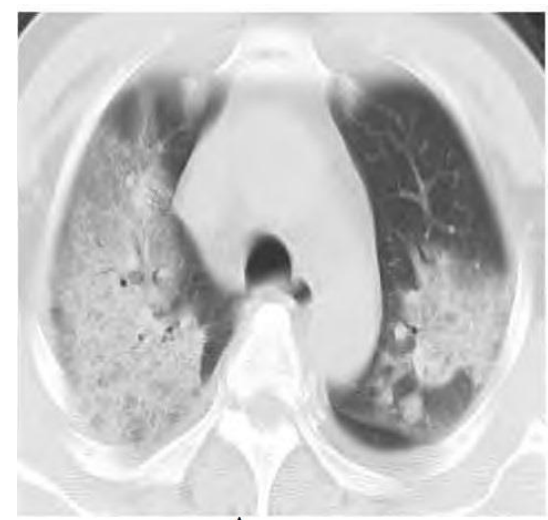

A

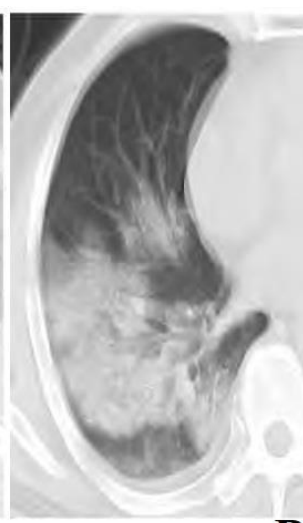

B

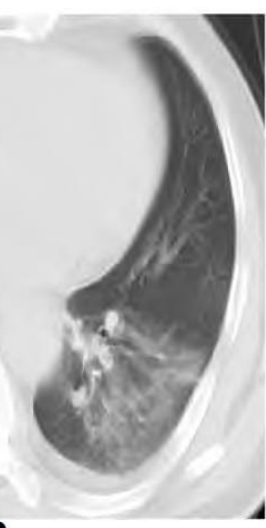

B

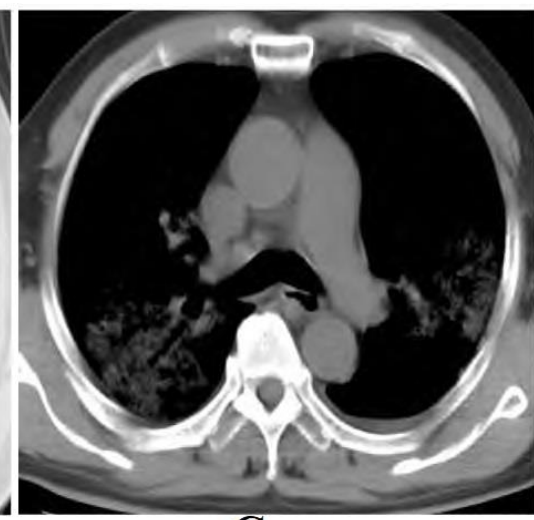

C

Figure VIII: COVID-19 patient 53 years old male. Have a history of close contacts. Had fever, cough, and chest tightness for 5 days and was admitted for an HRCT examination on the following day. A and B images of the lung window by CT scanning showing large patchy GGO shadows in the upper and lower lobes of both lungs, with a wide range of lesions, paving stone signs and consolidation shadows; C image a plain CT scan of the mediastinum window showing a small amount of effusion in the left pleural cavity

There were 4 patients with no apparent symptoms of aspiration tract HRCT in the lungs, and 2 patients with diarrhea had shown the only single or multiple small patches of ground glass opacity (GGO). Some lesions were distributed along with the broncho vascular bundle.

\section{Discussion}

Clinical Symptoms and Signs: The pathogens of COVID-19 and Severe Respiratory Syndrome Coronavirus (SARS-CoV) ${ }^{4}$ and the Middle East Respiratory Syndrome Coronavirus (MERS-CoV) ${ }^{5}$ belong to the Coronaviridae family. These are enveloped, positive-sense, single-stranded RNA viruses. 
Table 2: Summarize of All 24 Cases Information from This Study

\begin{tabular}{|c|c|c|c|c|c|}
\hline Items & $\mathrm{N}=\mathbf{2 4}$ & $\begin{array}{c}\text { Increase } \\
\text { n }(\%)\end{array}$ & $\begin{array}{c}\text { Normal } \\
\text { n }(\%)\end{array}$ & $\begin{array}{c}\text { Decrease } \\
\text { n }(\%)\end{array}$ & Percent \\
\hline Male & 15 & & & & 62.5 \\
\hline Female & 9 & & & & 37.5 \\
\hline$>60$ years age & 17 & & & & 71 \\
\hline \multicolumn{6}{|l|}{ Imaging Findings } \\
\hline $\begin{array}{l}\text { Lesions in the peripheral belt \& subpleural } \\
\text { areas in both lungs }\end{array}$ & 19 & & & & 79 \\
\hline Upper lobes of both lungs & 3 & & & & 13 \\
\hline Single lesions near the hilum & 2 & & & & 8 \\
\hline Lungs ground-glass opacity (GGO) & 22 & & & & 92 \\
\hline GGO with solid nodules & 14 & & & & 58 \\
\hline large-scale consolidation & 8 & & & & 33 \\
\hline Small amount of pleural effusion & 3 & & & & $13 \%$ \\
\hline Enlarged mediastinal \& white lung each & 2 & & & & $8 \%$ \\
\hline Grid-shaped fiber stripe shadow & 7 & & & & 29 \\
\hline \multicolumn{6}{|l|}{ Clinical Manifestation } \\
\hline Fever and cough combine & 3 & & & & 12.5 \\
\hline Elevated CRP & 24 & & & & 100 \\
\hline Asymptomatic & 4 & & & & 17 \\
\hline Diarrhea & 2 & & & & $8 \%$ \\
\hline Fever & 12 & & & & 50 \\
\hline cough & 3 & & & & 12.5 \\
\hline Respiratory symptoms & 18 & & & & 75 \\
\hline White blood cell count & & $6(25)$ & $6(25)$ & $12(60)$ & \\
\hline Lymphocyte count & & $5(21)$ & & $19(79)$ & \\
\hline Neutrophils cells count & & $10(42)$ & $9(37)$ & $5(21)$ & \\
\hline \multicolumn{6}{|l|}{ Epidemiological History of Cases } \\
\hline $\begin{array}{l}\text { History of staying in the affected area \& may } \\
\text { close contact with infected person }\end{array}$ & 18 & & & & 75 \\
\hline $\begin{array}{l}\text { No evident history of contact or stay in } \\
\text { epidemic area }\end{array}$ & 6 & & & & 25 \\
\hline
\end{tabular}

The virus has a strong ability to spread and can transmit in hospitals; the most common symptoms are fever and cough, and the rare symptoms are diarrhea and vomiting, which are more common in $m^{6-8}{ }^{6-8}$. In this study, the main symptoms of the patients are fever and cough (75\%), and the signs are more common in males $(62.5 \%)$. However, there are still some cases without obvious clinical symptoms; in a subclinical state, the diagnosis is difficult, easy to miss diagnosis or misdiagnosis, increases the chance of infection. DR images are easy to overlap, the rate of misdiagnosis is high, and the sensitivity and specificity are low. HRCT, on the other hand, has the advantages of high spatial resolution, feasible multi-plane reconstruction, and a three-dimensional display of lesions. Therefore, timely chest HRCT and virological examination of newly hospitalized patients are recommended with epidemiological support ${ }^{9}$.

\section{COVID-19 Image Signs}

Main Characteristics: In this group of cases GGO is $92.0 \%$ of COVID-19 patients with the most important lung manifestation, which are found in the peripheral and subpleural areas of both lungs. The leading cause of GGO may be due to the small virus particles. A large number of virus particles quickly gather at the end of the fine trachea and respiratory bronchioles of the alveolar epithelium. So, it is resulting in alveolar epithelial damage, vascular congestion, a small amount of alveolar exudate, and swelling of the tissues around the lung lobe. Furthermore, it mobilizes a large number of 
immune cells in the body's autoimmune system to form a leaflet-based image. As a result, single or multiple flaps and round GGO can be seen in the lung of COVID-19 patients, and the lesion range is relatively limited, mainly distributes in the peripheral lung and subpleural area. In some GGOs, the thickening and structural distortion of fine pulmonary vessels, the shadow of consolidation, and the shadow of high-density nodules are seen, as well as the widening of interlobular septa in some lesions. When large numbers of inflammatory cells infiltrate the alveolar space of COVID-19 patients, this can lead to increased alveolar swelling, bleeding, exudation, and forming a transparent membrane which covers with proteins and fibrin. Limited emphysema is caused by the widening distance of alveolar diffusion and the disturbance of ventilation function. The edges of the lesions of both lungs become wedge or fan-shaped due to enlargement of the lesion range and increase of the density. In the lesion, the widen interlobular septa shows a fine mesh shadow, which results in the appearance of wild paving stone. When the patient's pulmonary alveolar exudate is further increased, the virus causes Broncho Vascular bundle thickening, intrapulmonary interstitial lesion changes, and even high-density consolidation. In the short term, a large amount of virus accumulation can lead to the rapid progression of COVID-19 in patients, with diffuse lesions in both lungs, presenting as a typical "white lung." After receiving the correct treatment, the patient with COVID-19 get better; the chest CT mainly shows the reduction of the lesion area, the clear lesion boundary or the formation of fibrotic strips, the decrease in the density, and the complete disappearance of the lung lesions ${ }^{10}$.

In this group of patients, 4 has a history of close contact with confirmed cases or epidemic areas but no obvious clinical symptoms; 2 patients are diagnosed with diarrhea, but the nucleic acid test results are all positive. In these 6 patients, the chest HRCT shows only single or multiple small, thin patches of GGO with some lesions scattered along the subpleural or tracheal vascular tracts.

However, in the follow-up examination in the hospital, it is found that the lung lesions in these patients increases significantly and become dense. Some are showed air bronchi signs and "Paving stone sign" which are consistent with the image characteristics of viral pneumonia. Therefore, patients with occult infection with a clear epidemiological history, short-term follow-up for the re-examination of chest HRCT, is conducive to the detection of latent patients, early isolation, and treatment ${ }^{6}$.

Other signs: Among the 24 patients in this group, 3 cases are complicated with a small amount of pleural effusion.

However, viral pneumonia rarely causes pleural effusion, and it can only lead to inflammatory exudation and pleural effusion when the condition seriously involves with pleural effusion and causes pleurisy or reactive inflammation ${ }^{11}$. In this study, only 2 cases of mediastinal or bilateral hilar lymph node enlargement are similar to the chest imaging results of COVID-19 reported by Liu et $\mathrm{al}^{12}$. This may be due to the abnormal enlargement of the lymph nodes in the lung caused by lymphatic drainage through the mediastinum when the inflammation spreads to the lymph nodes ${ }^{13,14}$. The Author has summarized all the important information related to this study.

\section{Conclusion}

COVID-19 patients' chest HRCT is mainly manifested by early GGO and interstitial changes with distributed in the periphery of the two lung fields and the subpleural area. Typical features are extensive GGO in both lungs and maybe pulmonary consolidation in those with severe pleural effusion. Bilateral hilar and mediastinal lymphadenopathy are rare. Attention should be paid to the identification of this disease from viral pneumonitides such as cytomegalovirus (CMV) pneumonia, SARS, and MERS. The CT images of covid-19 have certain characteristics, which will facilitate the early diagnosis of the virus, early isolation, and treatment of covid-19 patients. Combined with clinical symptoms and epidemiological history of respiratory infections, chest HRCT has initial clinical diagnostic value.

[Availability of data and material: On reasonable request data portion will be available, but all data are not publicly available, because of patient's information.]

[Acknowledgments: Authors would like to thank all individuals to participate in the study.]

\section{References}

1. Zhu N, Zhang D, Wang W, et al. China Novel Coronavirus Investigating and Research Team. A novel coronavirus from patients with pneumonia in China, 2019. N Engl J Med. 2020;382(8):727-733.

2. Huang $\mathrm{C}$, Wang $\mathrm{Y}$, Li X, et al. Clinical features of patients infected with 2019 novel coronavirus in Wuhan, China. Lancet. 2020;395(10223):497-506. 
3. The General Office of the National Health Commission and the Office of the State Administration of Traditional Chinese Medicine. Diagnosis and treatment plan of pneumonia caused by COVID-19 (5 ${ }^{\text {th }}$ Edition) [EB/OL]. (2020-02-05) [2020-02$16]$.

http://www.nhc.gov.cn/xcs/zhengcwj/202002/3b09b894ac9b42 04a79db5b8912d4440.shtml.

4. Ksiazek TG, Erdman D, Goldsmith CS, et al. A novel coronavirus associated with severe acute respiratory syndrome. New England journal of medicine. 2003;348(20):1953-1966.

5. World Health Organization. Middle East respiratory syndrome coronavirus (MERS-CoV) [EB/OL]. [2019-12]. https://www.who.int/emergencies/mers-cov/en/

6. Wang $\mathrm{D}, \mathrm{Hu} \mathrm{B}, \mathrm{Hu} \mathrm{C}$, et al. Clinical characteristics of 138 hospitalized patients with 2019 novel coronavirus-infected pneumonia in Wuhan, China. Jama. 2020.

7. Holshue ML, DeBolt C, Lindquist S, et al. First case of 2019 novel coronavirus in the United States. New England Journal of Medicine. 2020.

8. Perlman S. Another decade, another coronavirus. In: Mass Medical Soc; 2020.

9. Chinese Medical Association of radiology. Radiological diagnosis of novel coronavirus-infected pneumonia: first edition of recommendations from Chinese Medical Association of Radiology experts [J/OL]. Chinese journal of radiology, 2020,54

10. Chen N, Zhou M, Dong X, et al. Epidemiological and clinical characteristics of 99 cases of 2019 novel coronavirus pneumonia in Wuhan, China: a descriptive study. Lancet. 2020;395(10223):507-513.

11. Koo HJ, Lim S, Choe J, Choi S-H, Sung H, Do K-H. Radiographic and CT features of viral pneumonia. Radiographics. 2018;38(3):719-739.

12. Liu Faming, Ding Huiling, Gong Xiaoming, et al. Chest CT manifestations and clinical features of novel coronavirus (COVID-19) [J/OL]. Radiology practice,2020.

13. Shah DP, Shah PK, Azzi JM, Chemaly RF. Parainfluenza virus infections in hematopoietic cell transplant recipients and hematologic malignancy patients: A systematic review. Cancer Lett. 2016;370(2):358-364.

14. Yoon H, Jhun BW, Kim SJ, Kim K. Clinical characteristics and factors predicting respiratory failure in adenovirus pneumonia. Respirology. 2016;21(7):1243-1250.

15. Jones RM, Brosseau LM. Aerosol transmission of infectious disease. Journal of occupational and environmental medicine. 2015;57(5):501-508 